\title{
Lesão Tumoral Primária do Pulmão em Adolescente
}

\section{Primary Pulmonary Tumor in an Adolescent}

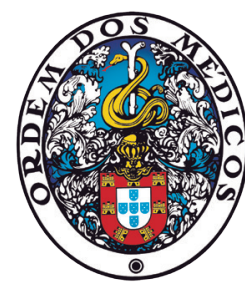

Luís RODRIGUES ${ }^{1,2}$, Rui DOMINGUES ${ }^{1}$, Diana AMARAL ${ }^{1}$, José CAVACO 1

Acta Med Port 2020 Jul-Aug;33(7-8):512-515 - https://doi.org/10.20344/amp.12690

RESUMO

Os tumores pulmonares primários em idade pediátrica são raros, existindo, contudo, uma multiplicidade de tipos histológicos, com diferentes abordagens terapêuticas. O tumor miofibroblástico inflamatório do pulmão representa $0,04 \%-1,2 \%$ de todos os tumores pulmonares, é mais frequente em crianças e adultos jovens e a sua etiologia é desconhecida. A apresentação clínica e radiológica é muito variável, pelo que o diagnóstico é difícil. Descrevemos o caso de um adolescente de 15 anos, com achado de nódulo pulmonar em radiografia de tórax realizada no contexto de infeção respiratória e cujo estudo etiológico revelou tratar-se de um tumor miofibroblástico inflamatório do pulmão. Foi realizada resseção atípica por toracoscopia videoassistida, verificando-se evolução favorável. Salientamos a raridade desta entidade, a necessidade de elevado índice de suspeição clínica e a marcha diagnóstica que levou ao esclarecimento definitivo e à sua resolução.

Palavras-chave: Adolescente; Granuloma de Células Plasmáticas; Miofibroma; Neoplasias do Pulmão

\section{ABSTRACT}

Primary lung tumors in the pediatric age group are rare, histologically diverse and have different therapeutic approaches. The inflammatory myofibroblastic tumor of the lung accounts for $0.04 \%-1.2 \%$ of all lung tumors, is more common in children and young adults and its etiology is unknown. The diagnosis is difficult as clinical and radiological findings are highly variable. We report a case of a 15-year-old adolescent who presented with a single pulmonary nodule on a chest radiograph, in the context of a respiratory infection, and whose etiological investigation revealed an inflammatory myofibroblastic tumor of the lung. Atypical resection was performed by video-assisted thoracoscopic surgery, with full recovery. We highlight the rarity of this entity, the need for a high suspicion index and the diagnostic investigation undertaken to reach a definitive diagnosis and a successful outcome.

Keywords: Adolescent; Granuloma, Plasma Cell; Lung Neoplasms; Myofibroma

\section{INTRODUÇÃO}

A maioria das massas pulmonares observadas em idade pediátrica resulta de processos inflamatórios, infeciosos ou reativos. ${ }^{1}$ Os tumores pulmonares primários são raros, existindo, contudo, uma multiplicidade de tipos histológicos, com diferentes abordagens terapêuticas. A sua apresentação clínica e radiológica mimetiza outras situações clínicas mais frequentes, o que pode levar a atrasos no diagnóstico e tratamento. ${ }^{2}$ Descrevemos o caso de um tumor miofibroblástico inflamatório do pulmão (TMIP) num adolescente, uma entidade rara e pouco conhecida, que exige elevado grau de suspeição para o diagnóstico.

\section{CASO CLÍNICO}

Adolescente de 15 anos, sexo masculino, antecedentes pessoais e familiares irrelevantes, avaliado em março de 2018 pelo médico assistente que, no contexto de infeção respiratória, solicitou radiografia de tórax. Esta revelou aumento focal de densidade homogénea, com morfologia arredondada, circunscrita, sobre o cruzamento dos arcos costais, na vertente externa do terço médio do hemitórax direito (Fig. 1). Cumpriu antibioticoterapia com amoxicilina e ácido clavulânico por suspeita clínica de sinusite aguda intercorrente e foi encaminhado para a consulta de Pneumologia Pediátrica de um hospital de nível III. Em abril de 2018 recorreu ao serviço de urgência por agravamento das queixas, com tosse, cefaleia, rinorreia e astenia e foi verifi- cada perda ponderal de $4 \%$ nos seis meses prévios. Foram negadas febre, anorexia, hipersudorese noturna, queixas ósseas, articulares, abdominais ou testiculares. Não foram observadas adenopatias cervicais, supraclaviculares, axilares ou inguinais, nem outras alterações ao exame objetivo. Perante este quadro, decidiu-se internamento para esclarecimento etiológico. Realizou estudo analítico que não revelou alterações (Tabela 1) e teste tuberculínico que foi anérgico. A tomografia computorizada de alta resolução e com contraste identificou uma formação nodular no segmento externo do lobo médio direito, poligonal, de contornos regulares, medindo $19,5 \times 16 \times 14,6$ mm de diâmetros transversal, ântero-posterior e longitudinal respetivamente, densidade sólida, levemente heterogénea, sem calcificações, sem componente de tecido adiposo e sem halo de densidade de vidro despolido do parênquima pulmonar adjacente (Fig. 2). Teve alta, sendo encaminhado para a consulta de Pneumologia Pediátrica. Dois meses após o internamento foi submetido a biópsia aspirativa transtorácica que identificou TMIP. Em equipa multidisciplinar foi decidida excisão da lesão, procedendo-se a resseção atípica do lobo médio por toracoscopia videoassistida, que decorreu sem intercorrências e com evolução posterior favorável. A avaliação histológica demonstrou miofibroblastos ALK positivos, AML fracamente positivos e CK AE1/AE3 negativos, com numerosos plasmócitos intralesionais, sem invasão

1. Unidade de Pneumologia Pediátrica. Área da Mulher, da Criança e do Adolescente. Hospital Dona Estefânia. Centro Hospitalar Universitário de Lisboa Central. Lisboa. Portugal.

2. Serviço de Pediatria. Departamento da Saúde da Mulher e da Criança. Hospital do Espírito Santo de Évora. Évora. Portugal.

$\triangle$ Autor correspondente: Luís Rodrigues. luisnorterodrigues@gmail.com

Recebido: 13 de agosto de 2019 - Aceite: 14 de outubro de 2019 | Copyright @ Ordem dos Médicos 2020 


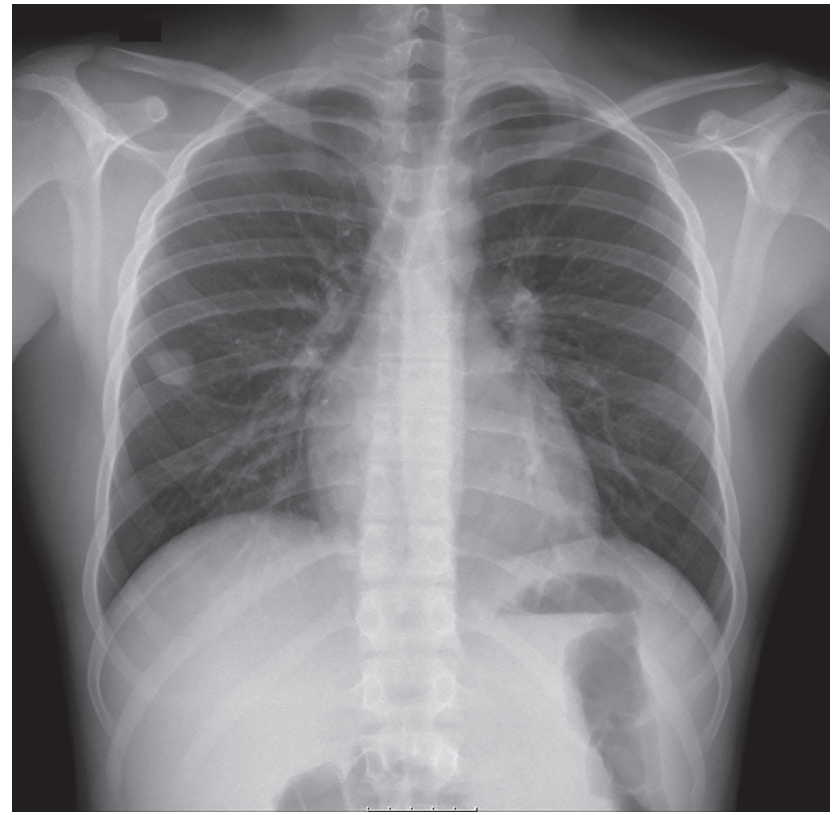

Figura 1 - Radiografia de tórax: nódulo pulmonar único no terço médio do hemitórax direito

vascular pelas células tumorais ou pleomorfismo nuclear $\mathrm{e}$ margem cirúrgica sem lesão (Fig. 3). Um ano após o diagnóstico, o adolescente mantém seguimento em consulta de Pneumologia Pediátrica, encontrando-se assintomático, com boa evolução ponderal e cicatricial, radiografia de tórax e espirometria sem alterações.

Tabela 1 - Estudo analítico efetuado em internamento

\begin{tabular}{lcc}
\hline Parâmetros & Resultados & Valores de referência \\
\hline Hemoglobina $(\mathrm{g} / \mathrm{dL})$ & 15,3 & $13-16$ \\
Leucócitos $/ / \mathrm{L})$ & 11650 & $6000-16000$ \\
Neutrófilos $(/ \mathrm{L})$ & 8250 & $1000-7000$ \\
Plaquetas $(/ \mathrm{L})$ & 246000 & $200000-550000$ \\
Proteína C reativa $(\mathrm{mg} / \mathrm{L})$ & 4,6 & $<5$ \\
Velocidade de sedimentação $(\mathrm{mm} / \mathrm{h})$ & 4 & $<11$ \\
Creatinina $(\mathrm{mg} / \mathrm{dL})$ & 0,79 & $0,1-0,36$ \\
Ureia $(\mathrm{mg} / \mathrm{dL})$ & 22 & $10,9-36,0$ \\
Sódio $(\mathrm{mEq} / \mathrm{L})$ & 139 & $136-145$ \\
Potássio $(\mathrm{mEq} / \mathrm{L})$ & 4,2 & $3,4-4,7$ \\
Cloreto $(\mathrm{mEq} / \mathrm{L})$ & 105 & $98-107$ \\
Cálcio $(\mathrm{mg} / \mathrm{dL})$ & 10,1 & $8,4-10,2$ \\
AST $(\mathrm{U} / \mathrm{L})$ & 14 & $14-35$ \\
ALT $(\mathrm{U} / \mathrm{L})$ & 10 & $8-22$ \\
GGT $(\mathrm{U} / \mathrm{L})$ & 11 & $7-21$ \\
Fosfatase alcalina $(\mathrm{U} / \mathrm{L})$ & 86 & $89-365$ \\
LDH $(\mathrm{U} / \mathrm{L})$ & 157 & $130-250$ \\
TSH $(\mu \mathrm{Ul} / \mathrm{mL})$ & 2,86 & $0,47-3,41$ \\
FT4 $(\mathrm{ng} / \mathrm{dL})$ & 0,86 & $0,89-1,37$ \\
FT3 $(\mathrm{pg} / \mathrm{mL})$ & 3,02 & $2,25-3,85$ \\
Alfafetoproteína $(\mathrm{ng} / \mathrm{mL})$ & 1,62 & $0,89-4,48$ \\
Antigénio carcinoembrionario $(\mathrm{ng} / \mathrm{mL})$ & 1,60 & $<5$ \\
\hline
\end{tabular}

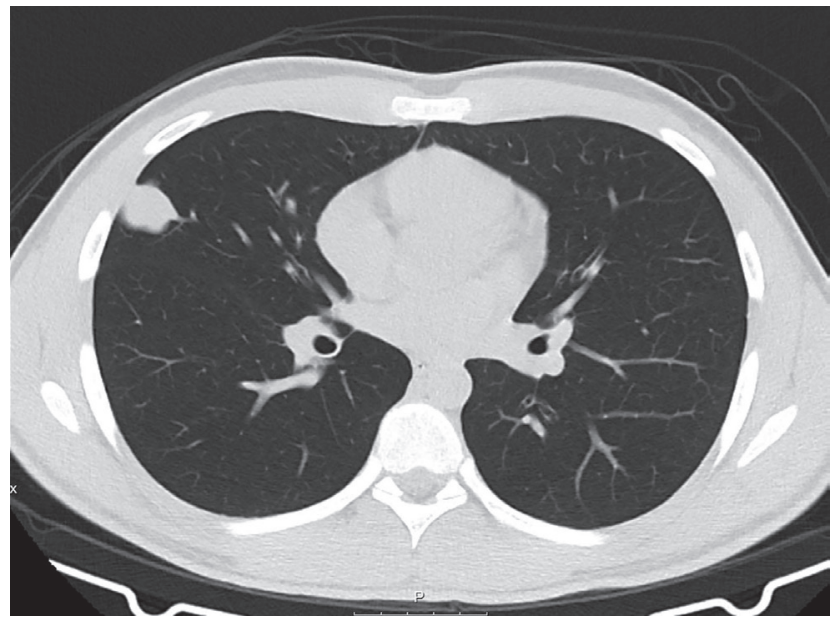

Figura 2 - Tomografia computorizada de alta resolução: nódulo pulmonar de densidade sólida, 19,5 × 16 × 14,6 mm, no segmento externo do lobo médio direito

\section{DISCUSSÃO}

O TMIP (também designado por pseudotumor inflamatório, granuloma de plasmócitos, histiocitoma fibroso, fibroxantoma ou xantogranuloma) representa $0,04 \%-1,2 \%$ de todos os tumores pulmonares. ${ }^{3}$ Pode surgir em qualquer idade, sem predomínio de género, sendo mais frequente em crianças e adultos jovens, onde corresponde a cerca de $20 \%$ de todos os tumores pulmonares primários e a $50 \%$ de todos tumores pulmonares benignos em idade pediátrica. ${ }^{4}$ 


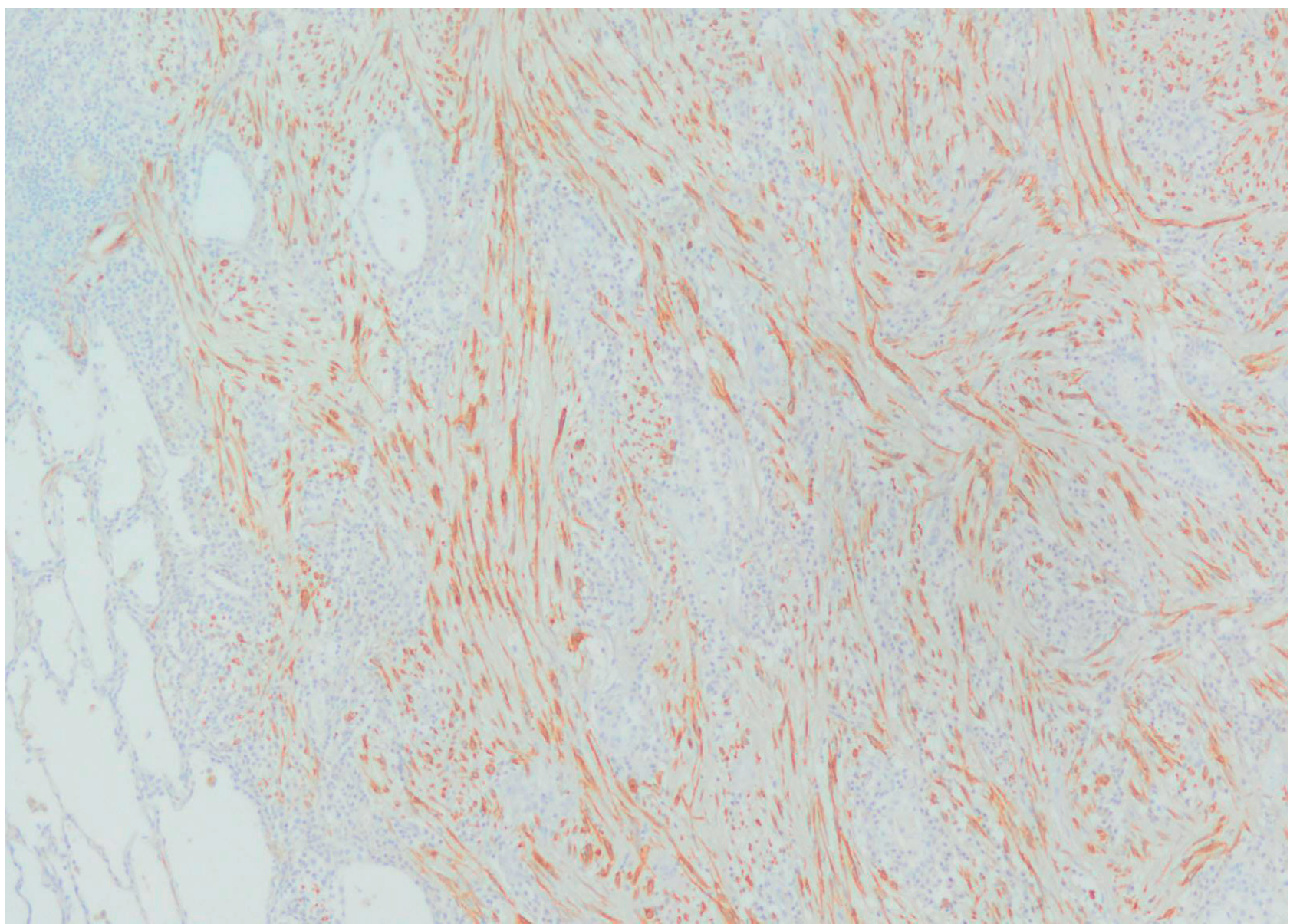

Figura 3 - Análise histopatológica: miofibroblastos ALK positivos, AML fracamente positivos e CK AE1/AE3 negativos, numerosos plasmócitos intralesionais

A etiologia é desconhecida, embora várias hipóteses sugiram a associação a mecanismos infeciosos (Mycobacteria, Mycoplasma, Nocardia, vírus Epstein-Barr e Herpes vírus 8), autoimunes e neoplásicos. ${ }^{5}$ Histologicamente são tumores caracterizados pela proliferação de miofibroblastos e células inflamatórias (linfócitos, plasmócitos e histiócitos), sendo descritos vários grupos histopatológicos, consoante o predomínio celular. ${ }^{5,6}$ As alterações do gene ALK (cinase de linfoma anaplásico), presentes em $50 \%$ dos TMIP, favorecem a carcinogénese por ativação constitutiva do recetor da tirosina cinase. ${ }^{7}$

A apresentação clínica é muito variável consoante a localização da lesão: os TMIP centrais são habitualmente diagnosticados mais cedo por obstrução da traqueia, carina ou brônquio principal, enquanto os TMIP periféricos se apresentam com sintomas inespecíficos, como tosse, febre, dispneia, anemia, perda ponderal, dor torácica e hemoptises, que condicionam um diagnóstico mais tardio. ${ }^{4-9}$

A radiografia de tórax demonstra habitualmente uma lesão nodular única, periférica, de contornos regulares e diâmetro variável (1,2 a $15 \mathrm{~cm})$, com predileção pelos lobos inferiores. Mais raramente apresenta-se sob a forma de lesões múltiplas ou como uma massa endobrônquica, estando indicada a investigação por broncoscopia. ${ }^{4,5} \mathrm{~A}$ tomografia computorizada é o exame de eleição, permite uma melhor caracterização das lesões e mostra uma atenuação e realce heterogéneos. ${ }^{6,7}$ Apesar de não ser usada tipicamente para a caracterização de lesões do parênquima pulmonar, a ressonância magnética pode ser vantajosa em lesões superiores a $3 \mathrm{~mm}$, com malformações vasculares ou inclusões de tecido adiposo. ${ }^{10} \mathrm{~A}$ tomografia por emissão de positrões tem uma sensibilidade elevada, mas baixa especificidade, estando indicada na avaliação da resposta ao tratamento em doentes não elegíveis para cirurgia. ${ }^{4,10}$

O diagnóstico diferencial inclui infeções, outros tumores pulmonares primários (benignos e malignos), metástases, malformações congénitas, aspiração de corpo estranho e sequestro pulmonar. ${ }^{4-6,10}$

A resseção cirúrgica é o tratamento de escolha em doentes com lesões únicas. ${ }^{11}$ Em caso de lesões múltiplas, irressecáveis ou em doentes com co-morbilidades importantes, podem ser consideradas outras modalidades terapêuticas como a corticoterapia, a radioterapia e a quimioterapia, com resultados variáveis..$^{12}$ Os inibidores da ALK (crizotinib) demonstraram-se eficazes em tumores ALK positivos, com respostas clínicas consistentes, embora limitadas a pequenas séries..$^{13,14}$ Que seja do nosso conhecimento, não existe na literatura nenhum relato da sua utilização no TMIP em idade pediátrica no nosso país.

O prognóstico é excelente quando a resseção cirúrgica 
é completa, com uma sobrevida aos cinco anos superior a $91 \%$. Os tumores ALK positivos têm melhor prognóstico que os negativos, que estão mais frequentemente associados a metástases e resistência à terapêutica. O seguimento deve ser prolongado pelo risco de recidiva e de transformação sarcomatosa. ${ }^{12}$

Embora não existam protocolos universalmente aceites para a abordagem de nódulos pulmonares únicos em idade pediátrica, a opção é frequentemente a de seguimento e vigilância. Apesar de na grande maioria dos casos esta ser a atitude com maior benefício em relação ao risco, há situações, como a do caso clínico descrito, em que as características da história do doente e da própria lesão, nos devem levar a maior intervenção na busca pelo diagnóstico e que exigem um elevado grau de suspeição. Os TMIP são difíceis de diagnosticar e, apesar de maioritariamente inócuos, a possibilidade de serem localmente invasivos e de sofrerem transformação maligna, reforça que o caminho tomado na investigação diagnóstica e abordagem cirúrgica deste caso foi o mais adequado.

\section{AGRADECIMENTOS}

Os autores gostariam de agradecer a Eugénia Pinto (Serviço de Anatomia Patológica do Hospital de São José, Centro Hospitalar Universitário de Lisboa Central), pelo seu

\section{REFERÊNCIAS}

1. Westra S, Brody A, Mahani M, Guillerman R, Hegde S, lyer R, et al. The incidental pulmonary nodule in a child. Pediatr Radiol. 2015;45:628-39.

2. Yu DC, Grabowski MJ, Kozakewich HP, Perez-Atayde AR, Voss SD, Shamberger R, et al. Primary lung tumors in children and adolescents: a 90-year experience. J Pediatr Surg. 2010;45:1090-5.

3. Içmeli OS, Alpay LA, Gundogus B, Turker H, Sen A. Inflammatory myofibroblastic tumor: a rare tumor of the lung. Eur Clin Respir J. $2014 ; 1$.

4. Camela F, Gallucci M, Palmo E, Cazzato S, Lima M, Ricci G, et al. Pulmonary inflammatory myofibroblastic tumor in children: a case report and brief review of literature. Front Pediatr. 2018;6:35.

5. Lai L, McCarville MB, Kirby P, Kao S, Moritani T, Clark E, et al. Shedding light on inflammatory pseudotumor in children: spotlight on inflammatory myofibroblastic tumor. Pediatr Radiol. 2015;45:1738-52.

6. Lakshmana DN, Newman B, Spottswood S, Narla S, Kolli K. Inflammatory pseudotumor. Radiographics. 2003;23:719-29.

7. Patnana M, Sevrukov A, Elsayes K, Viswanathan C, Lubner M, Menias C. Inflammatory pseudotumor: the great mimicker. AJR Am J Roentgenol. 2012;198:W217-27.

8. Fernandes A, Gil P, Resende J, Correia M, Tavares V, Valente J. Condensação pulmonar no jovem: a propósito de um caso clínico. Rev contributo através da análise histopatológica e seleção de imagens.

\section{PROTECÇÃO DE PESSOAS E ANIMAIS}

Os autores declaram que os procedimentos seguidos estavam de acordo com os regulamentos estabelecidos pelos responsáveis da Comissão de Investigação Clínica e Ética e de acordo com a Declaração de Helsínquia da Associação Médica Mundial.

\section{CONFIDENCIALIDADE DOS DADOS}

Os autores declaram ter seguido os protocolos do seu centro de trabalho acerca da publicação de dados.

\section{CONSENTIMENTO DO DOENTE}

Obtido.

\section{CONFLITOS DE INTERESSE}

Os autores declaram não ter nenhum conflito de interesses relativamente ao presente artigo.

\section{FONTES DE FINANCIAMENTO}

Não existiram subsídios ou bolsas que tenham contribuído para a realização do trabalho.

\section{Med Int. 2017;24:36-9.}

9. Brodlie M, Barwick SC, Wood KM, McKean MC, Welch A. Inflammatory myofibroblastic tumours of the respiratory tract: paediatric case series with varying clinical presentations. J Laryngol Otol. 2011;125:865-8.

10. Zapala MA, Ho-Fung VM, Lee EY. Thoracic neoplasms in children: contemporary perspectives and imaging assessment. Radiol Clin North Am. 2017; 55:657-76.

11. Dalton BG, Thomas PG, Sharp NE, Manalang MA, Fisher JE, Moir CR, et al. Inflammatory myofibroblastic tumors in children. J Pediatr Surg. 2016;51:541-4

12. Khatri A, Agrawal A, Sikachi RR, Mehta D, Sahni S, Meena N. Inflammatory myofibroblastic tumor of the lung. Adv Respir Med. 2018;86:27-35

13. Theilen T, Soerensen J, Bochennek K, Becker M, Schwabe D, Rolle $U$, et al. Crizotinib in ALK+ inflammatory myofibroblastic tumors-current experience and future perspectives. Pediatr Blood Cancer. 2018;65:1-8.

14. Mossé YP, Voss SD, Lim MS, Rolland D, Minard CG, Fox E, et al. Targeting ALK with crizotinib in pediatric anaplastic large cell lymphoma and inflammatory myofibroblastic tumor: a Children's Oncology Group Study. J Clin Oncol. 2017;35:3215-21. 